\title{
BETWEEN STRESS AND EVOLUTION - TEACHING AND LEARNING FRENCH FOR SPECIFIC PURPOSES IN ONLINE COURSES DURING COVID-19 PANDEMIA / ENTRE STRESS ET ÉVOLUTION - L'ENSEIGNEMENT/APPRENTISSAGE DU FOS À DISTANCE DANS UN CONTEXTE DE CONFINEMENT
}

\author{
Carmen Avram ${ }^{1}$, Felicia Constantin ${ }^{2}$ \\ 1 Université de Caen Normandie, CRISCO, Caen, France \\ ¿Université d'Oradea, Roumanie \\ elena.avram@unicaen.fr \\ fconstantin@uoradea.ro
}

\begin{abstract}
The pandemic calls into question the very functioning of humanity. In a world disturbed at all levels, education is inevitably suffering. The sudden passage without proper preparation exclusively to distance learning has changed the routines of all implied parties. This way of teaching and learning has pointed to many challenges, which led to new approaches, strategies, and methods as well as a need for a rapid adaptation to this unusual context. Our study, carried out during March and April 2021, proposed highlighting the representations and expectations of economics students (several specializations combined) at the beginning of their academic path. Through quantitative and qualitative analysis, the article identifies how the teaching and learning of the French language for affairs were influenced by the distance learning process imposed during the state of emergency and alert decreed by the government.

In the beginning, the students stressed their preference for the traditional learning paradigm (face-to-face courses, meeting with fellow students, the excitement of student life), even if they never crossed the doorsteps of an amphitheater. During the isolation period, despite the stress and challenges, they managed to discover the positive parts of online education, naturally provided by the tools used (unlimited access to courses, variety of teaching resources, freedom of access, the permanence of materials, video conferencing applications, etc.). As a result, the ideal learning environment is a combination of the two above-mentioned approaches that incorporate both worlds' advantages.
\end{abstract}

Keywords: distance teaching/learning; French for specific purposes; confinement; stress; videoconferencing; hybrid teaching.

JEL Classification: Z13

\section{Introduction}

La période comprise entre mars 2020 et mars 2021 représente une expérience hors normes pour tout le monde et relève de métamorphoses à tous les niveaux. Malgré la quasidésorientation au début du passage forcé à l'enseignement à distance, les chercheurs ont essayé d'en identifier les changements et les défis; les praticiens ont tenté d'en identifier les obstacles et les blocages dans l'activité didactique, les théoriciens ont investigué le phénomène afin de proposer des solutions pragmatiques ; les enseignants et les élèves/ les étudiants ont essayé de s'y adapter au fur et à mesure des jours et des semaines de cours. Durant cette période, beaucoup d'études ont été publiées, notamment dans le domaine de l'économie, de la santé ou de la psychologie, mais aussi en didactique et en pédagogie. De façon générale, ces études ont mis en évidence les problèmes et les blocages de toute sorte, induits par cette crise mondiale ; elles ont décrit des situations et des expériences durant la période de confinement et ont proposé des solutions et des pistes rapides d'amélioration des pratiques. 
Notre article se propose d'interroger l'expérience vécue par des étudiants roumains en première année d'études économiques, qui étudient le français en tant que première langue étrangère, dans le cadre de leurs spécialisations.

Nous nous proposons d'identifier les représentations, les attentes et les suggestions de ces étudiants par rapport à l'expérience vécue durant la période imposée d'enseignement à distance et plus particulièrement par rapport au cours de français en ligne.

\section{Contexte}

La pédagogie universitaire et l'utilisation des ressources numériques questionnent depuis plusieurs années l'enseignement supérieur. De même, la conception des cours à distance pour des publics en formation initiale ou continue, respectivement l'essor des plateformes permettant d'accueillir ces cours préoccupent le milieu universitaire, car selon Endrizzi (2012 : 11) « avec les technologies numériques, ce sont les opportunités d'apprendre qui se démultiplient », le numérique étant un vecteur innovateur d'enseignement et d'apprentissage. Malgré différents projets d'enseignement à distance développés au niveau européen depuis les années 2000, la crise de la COVID-19 et le passage massif à distance ont constitué un grand défi pour la communauté universitaire, tout comme pour l'enseignement en général et à tous les niveaux.

\subsection{Le confinement pédagogique : une expérience hors normes}

Des études ont été publiées durant la période du confinement en Europe et ailleurs et ont souligné la difficulté engendrée par l'adaptation brutale, rapide et surtout obligatoire à l'enseignement et à l'apprentissage à distance. Notons ici le numéro de juillet 2020 de la revue "Recherches et éducations» ou bien le numéro 9/2020 du "Journal des psychologues », consacrés intégralement à la crise de la COVID-19.

Descamps et al. (2020) s'intéresse aux aspects de la continuité pédagogique en Belgique francophone et identifie plusieurs difficultés comme l'aspect technique et le manque d'outils adaptés, l'absence de formation aux outils numériques, le manque d'accès à l'outil informatique ou à l'internet; du côté pédagogique, les difficultés relèvent de l'absence de feedback, extrêmement démotivant pour les apprenants, ainsi que de la difficulté de gestion des grands groupes d'étudiants, due à l'absence d'interaction et au manque d'outils adaptés. Les auteurs soulignent ainsi que le numérique intégral, notamment dans ce contexte contraint, entraine des difficultés à plusieurs niveaux, puisque tous les enseignants et les apprenants / étudiants « ne sont pas égaux face aux tâches numériques proposées » (Descamps et al. 2020).

En prenant comme exemple le milieu universitaire, Denny (2020) réalise une étude auprès d'un groupe d'étudiants de deuxième année de licence en Sciences de l'éducation à l'université de Strasbourg. L'auteur constate que la difficulté majeure apportée par cette crise consiste en l'absence de toute anticipation face à cette situation inédite et la perte de repères déterminée par la transformation des routines : le rôle de l'enseignant, ainsi que celui de l'étudiant, changent complètement, car l'enseignement repose intégralement sur des plateformes numériques. La figure d'autorité de l'enseignant est désacralisée et devient " plus fraternelle, plus humaine », car " un contrat tacite se dessine avec l'enseignant qui fait l'effort de retravailler ses cours et qui s'implique dans une dimension affective ressentie comme plus vraie » (Denny, 2020). D'autre part, l'étudiant participe de façon plus directe à l'apprentissage (car il est davantage sollicité et mis en situation d'autonomie), s'implique aussi dans cette dimension psychoaffective dans les relations avec l'enseignant et attribue un sens et une valeur aux tâches universitaires.

Dans ce changement rapide et souvent brutal des pratiques, de nombreux points sont concordants avec les apports précurseurs de Dieumegard et Durand (2005) et de Massou 
et Lavielle-Gutnik (2017). On retrouve ainsi chez eux l'abandon du tutorat et de la relation face à face de l'enseignant et de l'étudiant en échange des espaces institutionnalisés comme la plateforme Moodle. La communication directe est remplacée par la communication différée lorsque les cours sont donnés en modalité asynchrone et les étudiants sont obligés constamment de se construire de nouveaux repères. La communication et les liens sociaux sont mis à l'épreuve dans le milieu universitaire comme dans toute la société et certains auteurs comme Conrath et Ouazzani (2020) mettent en débat les effets néfastes de cette distance prolongée, ils se questionnent sur l'utilité réelle et à long terme des outils numériques qui se développent sans cesse et qui ont tendance évidente à se substituer au " présentiel » et à engendrer de nouveaux modes d'interaction. Mais, au-delà des difficultés, il faut souligner également l'apport majeur de ce changement des routines qui suscite aussi une plus forte capacité d'adaptation, de création et de conception d'activités et de scénarios pédagogiques. Guironnet (2020) souligne dans ce sens que « par la force des choses, le virtuel devient réalité tangible et ce quotidien permet de créer de nouveaux scénarios pédagogiques, de nouveaux cadres de pensée, de fonctionnement avec une interactivité et une créativité différente de "l'école d'avant » ».

C'est en réfléchissant à tout cet ensemble de points négatifs et de points positifs - vécus au quotidien par notre expérience d'enseignement et soulignés par les différentes recherches - que nous avons entamé cette étude. Avant de décrire l'enquête proposée auprès de nos étudiants, nous allons décrire le contexte institutionnel et pédagogique dans lequel elle se situe.

\subsection{Typologie de l'enseignement dans une université roumaine avant la pandémie due à la COVID-19}

En mars 2021, à la suite de la proclamation de la pandémie par l'OMS (https://www.who.int/fr/news/item/29-06-2020-covidtimeline), le président de la Roumanie a émis le décret qui a proclamé l'état d'urgence sur le territoire de la Roumanie. Le lockdown général a continué avec l'état d'alerte médicale renouvelé à plusieurs reprises, ce qui a eu le même effet : l'interdiction d'aller à l'école. Les élèves de lycée se sont retrouvés dans une situation exceptionnelle, qui les a obligés de suivre le deuxième semestre de l'école sous une forme non expérimentée jusque-là. Le travail en ligne s'est révélé être un quasi-échec, dévoilant des failles profondes dans la formation des enseignants, jamais exposés à ce type d'activité didactique (les rares exceptions ne peuvent pas être évoquées en tant que régularité).

Au niveau des élèves, le choc a été tout aussi puissant, parce que les habiletés de travailler sur des dispositifs électroniques et sur des réseaux sociaux étaient loin de ce qui leur fallait pour valoriser leurs connaissances de manière systématique, dans le cadre de leurs étudies obligatoires. Les élèves ayant obtenu le diplôme de baccalauréat dans un contexte hors normes en 2020 , sont devenus au mois d'octobre de la même année la première génération d'étudiants n'ayant jamais franchi les seuils d'un amphithéâtre.

Presque chaque université roumaine fournit une double orientation: cours de jour en présentiel respectivement e-formation à distance. Entre ces formes d'enseignement, il existe de nombreuses similarités de fond (au niveau des matières scolaires, des contenus, de l'évaluation, des diplômes) et quelques différences de forme (en relation avec la subvention financière, les bénéfices financiers tels que les bourses sociales et de performances, la forme d'organisation didactique et les instruments associés).

L'e-learning existe alors depuis longtemps, il n'est pas apparu une fois la pandémie déclarée. Cette formation est un choix assumé par des personnes qui ne peuvent pas se dédier exclusivement aux études, des personnes qui choisissent de compléter leur formation pour mieux s'adapter au marché du travail, qui ont déjà un emploi et veulent le maintenir ou se qualifier pour avancer dans la carrière. Eux, ils assument complètement les éventuels désavantages et prennent pour avantage exactement ce qui pourrait être perçu comme 
désavantage par les étudiants qui se dédient exclusivement aux études : la dispense de la présence physique hebdomadaire aux cours, l'absence du contact permanent avec l'enseignant et les collègues et surtout ce que le jeune a investi dans la projection imaginaire de la vie d'étudiant ou du statut d'étudiant.

Pourquoi les études à distance en confinement ne sont-elles pas identiques avec l'e-learning déjà en place ? Tout simplement parce qu'elles s'accompagnent de l'obligation et de la contrainte de faire autrement de ce que les attentes des jeunes apprenants auraient laissé croire. En plus, on associe inconsciemment une dimension d'interactivité ou d'interaction comme garantie du succès de l'apprentissage ; or, l'expérience le prouve que le plus souvent, malgré leur présence physique dans la salle de cours, les étudiants posent très rarement des questions et sont assez loin d'une implication totale dans la vie étudiante.

\section{L'enquête proposée aux étudiants roumains en sciences économiques}

Afin de saisir de façon plus précise les difficultés ressenties par ces étudiants novices, durant leur première année universitaire et dans un contexte si peu propice à leur formation en tant qu'étudiants, nous avons conçu un questionnaire qui a été complété en ligne. Une autre étude a déjà été réalisée dans la même faculté, sur un public plus hétérogène comme niveau d'études et qui étudie l'anglais. Cette étude (Sim et Pop, 2020) s'est proposé de décrire certaines analogies et différences entre les cours en face à face et en direct en ligne. Elle a visé principalement les enseignants et a mis en évidence les conclusions suivantes : les élèves préfèrent le type d'apprentissage traditionnel en face à face, tandis que l'enseignant reste un guide pour les étudiants, un facilitateur très créatif et innovant, apportant des idées nouvelles.

\subsection{Méthodologie}

En ce qui nous concerne, deux études majeures se trouvent à la base de notre réflexion et de la construction du questionnaire. D'une part, l'étude de Drissi (2010) qui met en évidence les caractéristiques des questions en ligne, dans un environnement vidéographique synchrone et qui souligne la nécessité de savoir alterner de façon adéquate les cadres communicationnels pour mener une interaction d'enseignement/apprentissage en ligne. Cette étude nous a inspiré la série de questions autour de la communication en classe de langue, du ressenti des étudiants par rapport aux occasions de communiquer, de s'exprimer de façon directe, dimension essentielle de l'apprentissage d'une langue étrangère. D'autre part, la thèse de Quentin (2008) concernant l'accompagnement tutoral dans les formations en ligne et les catégories d'interventions tutorales, organisées autour des composantes pédagogique, socio-affective, organisationnelle et technique.

\subsection{Questionnaire et public cible de l'étude}

Un questionnaire avec une quarantaine de questions a été distribué aux étudiants en première année qui étudient à la Faculté des Sciences Economiques d'Oradea, une ville en pleine expansion située à la frontière ouest de la Roumanie. Plusieurs spécialisations ont été visées : Comptabilité, Finances et Banques, Marketing, Management et Économie du Commerce, du Tourisme et des Services. Ces étudiants ont un cours et un séminaire de langue étrangère toutes les deux semaines dans le premier semestre, respectivement un cours toutes les deux semaines et un séminaire hebdomadaire pendant le deuxième semestre. À la fin de chaque semestre, ils passent un examen; la note finale se compose de la moyenne des évaluations du cours et du séminaire, l'assiduité étant prise en compte. Au moment de l'investigation, les sujets sont en deuxième semestre, ce qui laisse supposer qu'ils peuvent s'exprimer par rapport à plusieurs aspects de leur vie universitaire.

65 étudiants sur 105 qui étudient le français en première année ont répondu aux questions, ce qui représente $62 \%$ du total inscrits en français et environ $80 \%$ si l'on prend en compte 
l'assiduité. Au total, 54 étudiantes et 11 étudiants, avec un âge moyen de 18-19 ans. Parmi les répondants, 16 étudiants travaillent pendant leurs études.

En ce qui concerne les compétences en français, 2 étudiants sont des débutants et ils n'ont pas étudié le français avant leur cursus universitaire, alors que les autres se partagent la plage de manière relativement équilibrée : $24,6 \%$ ont étudié entre 2 et 4 ans, $18,5 \%$ entre 4 et 6 ans, 33,8\% des étudiants ont déjà étudié le français depuis au minimum six ans, tandis que $20 \%$ l'étudient depuis plus de 8 ans.

\section{Résultats obtenus}

Nous allons présenter les résultats de cette enquête selon le modèle proposé par Quentin (2008) et exploité également par Descamps et al. (2020).

\subsection{La composante technique}

Cette composante met en évidence la présence ou le manque de compétences numériques, l'accès à l'outil informatique et à l'internet ou la fracture numérique. Nous avons interrogé également la typologie des outils numériques utilisés par les étudiants pour les cours en ligne et les cours de français plus précisément.

Les dispositifs les plus utilisés sont le téléphone (47 étudiants), l'ordinateur fixe (44 étudiants), l'ordinateur portable (10 étudiants) ou la tablette (2 étudiants). Certains utilisateurs varient les supports d'une séance à l'autre.

Selon Guironnet (2020), l'équipement utilisé influence beaucoup la qualité et la quantité du travail fourni par les étudiants surtout en ce qui concerne la lecture des textes et la production écrite. De cette façon, pour notre public cible, la composante technique prise en compte ne devrait pas influencer de façon négative l'expérience des cours en ligne, car les étudiants bénéficient de bonnes conditions de travail. Mais l'utilisation du téléphone restreint de manière significative les tâches didactiques qui peuvent être réalisées.

\subsection{La composante organisationnelle}

Nous nous sommes intéressées au temps accordé par nos étudiants aux différents cours en ligne et plus précisément au cours de français. La plus grande partie des étudiants consacrent entre $50 \%$ et $95 \%$ de leur temps aux études en général, alors que pour le cours de français ils consacrent majoritairement entre $50 \%$ et $75 \%$ du temps qu'ils estimeraient nécessaire pour obtenir de la performance. La nouvelle expérience introduit la famille comme facteur en présence, ce qui peut troubler l'implication totale et la concentration aux cours en ligne.

\subsection{La composante pédagogique}

Le mode en ligne est considéré comme étant plus efficace par 32,3\% des répondants, par rapport au mode face-à-face. Ils argumentent leur point de vue en mentionnant des éléments comme l'accès à plusieurs ressources considérées comme " aide » (dictionnaire online, sites, instruments de travail) ; la possibilité de revenir sur un certain document, chargé sur la plateforme par le professeur ; la préférence pour le travail avec l'ordinateur ; l'opportunité d'apprendre plusieurs choses nouvelles; la commodité et le confort d'étudier depuis chez soi ; la facilité de chercher l'information; la possibilité de se renseigner en temps réel sur plusieurs sites ; la chance de travailler à son rythme.

L'argumentation en faveur du face-à-face est fondée sur les aspects suivants, déterminants pour presque $70 \%$ des étudiants interrogés : l'importance de l'interaction avec l'enseignant et les collègues ; l'incapacité de l'ordinateur de capter l'attention ; la difficulté d'apprendre seul.

Les étudiants en première année ont des attentes, mais ils n'ont pas d'expérience. 97\% 
d'entre eux sont étudiants pour la première fois, donc ils ne connaissent pas les particularités d'une formation universitaire en face à face. II manque par conséquent de leur argumentaire des éléments tels que ceux cités dans l'étude de Denny (2020) : l'amusant, le récréatif, l'intention de ne pas décevoir le professeur, la logique du don et contre-don, l'accent sur le support et non pas sur l'affectif, la privatisation des relations, l'accent sur l'analyse et l'interprétation en défaveur de la mémorisation des cours, la capacité attentionnelle, l'approfondissement par soi-même, la pression du copié-collé, la fonction cathartique du savoir appris, le sentiment de culpabilité pour toute forme d'oisiveté, l'intensité du rythme et du travail en ligne par rapport à la lenteur ressentie en face à face, etc.

L'activité didactique se déroule sur une plateforme Moodle, sur laquelle sont enrôlés tous les étudiants, dans des pages des disciplines scolaires correspondant à leur formation spécifique. Ils ont accès à tous les matériels, chargés à raison hebdomadaire par chaque enseignant. Lors du cours une session de chat et ouverte, qui permet une communication écrite directe.

L'un des grands minus de cette version de plateforme utilisée dans l'université analysée est l'absence de la facilité audio et vidéo, qui n'est pas intégrée dans les options d'usages. Pour pallier ce manque, les enseignants choisissent d'ouvrir des séquences de communication audio-vidéo, utilisant des applications de visioconférence telles que : Zoom, Skype, Google Meet ou Teams. Celles-ci permettent de communiquer à l'oral, de partager l'écran, de transférer aux étudiants l'option de partage de l'écran. Pour Guironnet (2020), le « lever la main » pour avoir la parole et le micro rendu audible par la manipulation de l'enseignant ou de l'étudiant sont des éléments facilitateurs des plateformes, qui dynamisent le dialogue.

Malgré les difficultés soulignées, les étudiants identifient également l'apport positif de cette période et il est rassurant de découvrir leurs réponses concernant notamment l'intégration des nouvelles technologies dans l'acte d'apprendre (52,3\% des étudiants) et une meilleure gestion du temps de travail $(41,5 \%$ des étudiants).

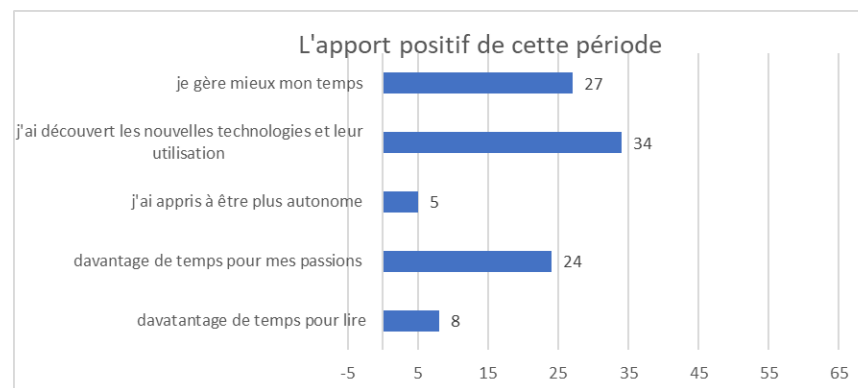

Figure 1. Qu'est-ce que cette période vous a-t-elle apporté de positif ?

Source : Questionnaire mars-avril 2021

Nous avons également souhaité interroger les avis et les préconisations de nos étudiants par rapport à l'avenir des cours universitaires. Ainsi, s'avère-t-il intéressant de remarquer d'une part le nombre d'indécis (19 étudiants, représentant $29,2 \%$ de l'effectif total), ce qui est révélateur de leur manque d'expérience, car débutants dans le monde universitaire. Et, d'autre part, les préconisations de 26 d'entre eux (40\% de l'effectif) pour un enseignement hybride. Très peu d'étudiants (6) envisagent un enseignement intégralement en présentiel, alors que l'enseignement à distance est plébiscité par 14 étudiants (21,5\%). II est ainsi évident que le « distanciel » est de plus en plus envisagé par nos étudiants et considéré comme étant une modalité d'enseignement/apprentissage nécessaire et incontournable désormais. 
L'avenir des cours universitaires

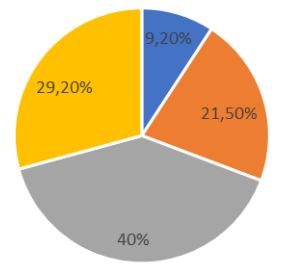

- $100 \%$ en présentiel $\quad 100 \%$ en ligne $\quad$ "hybride $\quad$ " je ne sais pas

Figure 2. Quel avenir pour les cours universitaires ?

Source : Questionnaire mars-avril 2021

\subsection{La composante socio-affective}

Concernant cette composante, notre intérêt s'est porté sur le vécu durant les cours de français : les étudiants ont eu ou bien l'impression d'être " bien accompagnés " (47 étudiants, représentant $72,3 \%$ de l'effectif interrogé) ou bien « isolé » (18 étudiants, à savoir $27,7 \%$ du total). Les sentiments vécus par rapport à ce cours sont partagés entre l'indifférence (1 étudiant), le stress (8), le manque de motivation (4), la relaxation (19), la curiosité (44), la motivation (26), l'intérêt (1), l'absence de sentiment (5). La question portant sur l'évaluation du stress ressenti et de ses causes éventuelles, nous a offert des réponses intéressantes : l'horaire du cours est perçu comme un élément stressant par 25 étudiants, l'absence d'interaction avec l'enseignant par 18 étudiants et l'absence de la communauté de collègues par 13 étudiants. Alors que pour 24 étudiants, rien ne semble constituer un élément déclencheur de stress.

Le graphique ci-dessous regroupe les réponses obtenues à la question concernant les manques ressentis par rapport au cours de français : nous remarquons ainsi que ce qui manque le plus à nos étudiants est la présence effective dans une salle de classe (notée par le maximum par 35 étudiants), suivi par le contact direct avec le professeur et le contact direct avec les collègues.

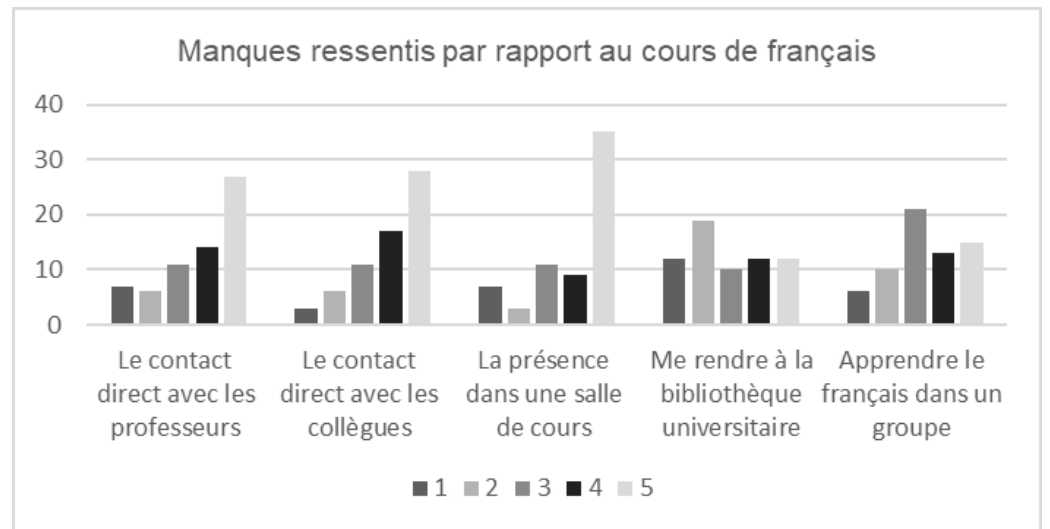

Figure 3 : Qu'est-ce qui vous manque le plus?

Source : Questionnaire mars-avril 2021

Cette composante a un impact majeur sur l'approche individuelle des participants au cours, parce que, selon Dugas et al. (2020), « l'être humain n'agit pas comme un robot calculateur et froid, que ses caractéristiques - dont ses émotions - troublent la logique prédictive ». 
L'impact du confinement est extrêmement inquiétant et l'ample étude de Bourion-Bédès, S. et al. (2021) démontre que l'anxiété lors du verrouillage en raison de l'épidémie de COVID19 peut atteindre plus de la moitié des étudiants d'une région touchée par la maladie.

5.

Conclusions

L'expérience de l'apprentissage en confinement est apparue au commencement comme une expérience déstabilisante. Au fur et à mesure, l'obligation et la contrainte se sont amoindries, pour faire face à l'habitude et même à la performance. Le travail en ligne représente un défi à tous les niveaux et tous les acteurs impliqués sont amenés à s'y plier. Les enseignants se sont familiarisés avec les facilités des plateformes didactiques et avec les instruments de type visioconférence. Les étudiants en première année, interrogés dans notre questionnaire ont vécu des changements en matière de leur comportement adaptatif et de leurs attentes. Ils sont conscients de l'appauvrissement à propos des expériences sensorielles : le contact avec les collègues, l'expérience de l'amphithéâtre, de l'audition d'un cours magistral avec tout le cérémonial symbolique, l'expérience de la pause, de l'activité par proximité personnelle. Mais ils commencent à constater que l'on peut fonctionner de manière optimum, en attendant l'ouverture des portes physiques de l'université.

Les étudiants qui ont subi le baptême de l'université à travers un écran ne peuvent pas faire des analyses consistantes relatives à la situation donnée. Ils se rapportent seulement à leur expérience de lycée et les arguments principaux invoqués tournent autour de la proximité physique étudiant - étudiants - enseignant - enseignants et de leurs rapports réciproques. II y a tout un imaginaire construit autour du moment où l'on devient / l'on est étudiant. II y a des expériences communes et des souvenirs qui se capitalisent. Or, derrière un écran, ce tableau perd de contours.

Si l'accent est mis dans certaines recherches sur la continuité pédagogique (Wagnon, 2020), dans notre cas on parle de l'instauration dans un contexte pédagogique inaccoutumé, car vécu pour la première fois. D'ici l'inquiétude initiale de la part des enseignants et des étudiants, amenés à une harmonisation et une optimisation de l'activité commune et individuelle.

II semblerait que cette étape de passage général accéléré au numérique aurait un vrai effet de levier, pour d'aboutir à une société apprenante, qui mise sur l'éducation tout au long de la vie, car « inventer l'éducation supérieure du XXle siècle suppose de passer à une pédagogie active, intégrant les apports du numérique » (Tadei et al., $2017: 6$ ).

\section{Références}

1. Bourion-Bédès, S. et al. (2021) "Psychological impact of the COVID-19 outbreak on students in a French region severely affected by the disease: results of the PIMS-CoV 19 study", Psychiatry

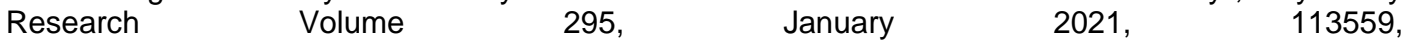
https://www.sciencedirect.com/science/article/pii/S0165178120332200?via\%3Dihub, consulté le 16 février 2021.

2. Conrath P. and Ouazzani M. (2020) "Les Relations, à bonne distance ? ", Le Journal des psychologues, 2020/9 $\mathrm{n}^{\circ}$ 381, ISSN 0752-501X; https://www.cairn.info/revue-le-journal-despsychologues-2020-9-page-3.htm, consulté le 16 février 2021.

3. Denny, J.-L. (2020) « Le confinement pédagogique : de la pandémie à l'expérience d'apprentissage des étudiants ", Recherches \& éducations [En ligne], HS | Juillet 2020, DOI: https://doi.org/10.4000/rechercheseducations.10252, consulté le 16 février 2021.

4. Descamps, S., et al. (2020) "Perception de la continuité pédagogique des participants à un webinaire sur l'apprentissage à distance dans un contexte de confinement ", Recherches \& éducations [En ligne], HS | Juillet 2020, DOI : https://doi.org/10.4000/rechercheseducations.10562, consulté le 16 février 2021.

5. Drissi, S. (2010) « Quelles questions poser à l'apprenant de langue en ligne? » Revue internationale des technologies en pédagogie universitaire / International Journal of Technologies in Higher Education, 7 (2), 60-74. https://doi.org/10.7202/045249ar, consulté le 16 février 2021. 
6. Dugas, É. et al. (2020) « Le coronavirus à l'épreuve des stratégies interactionnelles. Entre dilemmes et enjeux sociétaux ", Recherches \& éducations [En ligne], HS | Juillet 2020, DOI: https://doi.org/10.4000/rechercheseducations.10387, consulté le 16 février 2021.

7. Endrizzi, L. (2012) «Les technologies numériques dans l'enseignement supérieur, entre défis et opportunités », Dossier d'actualité Veille et Analyses, $\mathrm{n}^{\circ} 78$, octobre. En ligne: http://ife.enslyon.fr/vst/DA/detailsDossier.php?parent=accueil\&dossier=78\&lang=fr, consulté le 16 février 2021.

8. Guironnet, G. (2020) « Du réel au virtuel : une éducation par le 3e corps », Recherches \& éducations [En ligne], HS | Juillet 2020, DOI : https://doi.org/10.4000/rechercheseducations.10291, consulté le 16 février 2021.

10. Massou, L. et Lavielle-Gutnik (2017) Enseigner à l'université avec le numérique. Savoirs, ressources, médiations, De Boeck Supérieur.

11. OMS (2021) Chronologie de l'action de l'OMS face à la COVID-19 https://www.who.int/fr/news/item/29-06-2020-covidtimeline

12. Quintin, J.-J. (2008) «Accompagnement d'une formation asynchrone en groupe restreint : modalités d'intervention et modèles de tutorat », Sciences et Technologies de I'Information et de la Communication pour l'Éducation et la Formation, volume 15, 2008. ÉPAL : Échanger Pour Apprendre en Ligne. pp. 89-123; doi: https://doi.org/10.3406/stice.2008.981, consulté le 16 février 2021.

13. Descamps, S. et al. (2020) «Perception de la continuité pédagogique des participants à un webinaire sur l'apprentissage à distance dans un contexte de confinement ", Recherches \& éducations [En ligne], DOI : https://doi.org/10.4000/rechercheseducations.10562, consulté le 16 février 2021.

14. Sim M-A, Pop, A.-M. (2020) "In the defense of teachers in times of crises - thoughts, idea, opinions regarding teachers", Analele Universitatii din Oradea, Economic Sciences TOM XXIX, pp. 390-399, http://anale.steconomiceuoradea.ro/en/wp-content/uploads/2021/02/Anale-AUOES Issue-2-din2020.pdf, consulté le 16 février 2021.

15. Taddei, J-F., Becchetti-Bizot, C. et Houzel, G. (2017) Vers une société apprenante, Rapport sur la recherche et développement de l'éducation tout au long de la vie. Paris : Ministère de l'Éducation nationale, de l'enseignement et de la recherche. Disponible en ligne https://www.education.gouv.fr/vers-une-societe-apprenante-rapport-sur-la-recherche-etdeveloppement-de-l-education-tout-au-long-de-5843, consulté le 16 février 2021.

17. Wagnon, S. (2020) « La continuité pédagogique: méandres et paradoxes en temps de pandémie ", Recherches \& éducations [En ligne], HS | Juillet 2020, DOI : https://doi.org/10.4000/rechercheseducations.10451, consulté le 16 février 2021. 